II.

\title{
Gebirgsbildung und Massengesteine in der Kordillere Südamerikas.
}

Von

G. Steinmann in Bonn.

\section{Die Kordillere.}

Mit 11 Textfiguren.

Auf dem schmalen, langgestreckten Streifen der Erdrinde, der heute von der Kordillere Südamerikas eingenommen wird, haben zu wiederholten Malen gebirgsbildende Vorgänge verschiedener Art Platz gegriffen, und ebenso ist diese Gegend mehrfach der Schauplatz sowohl vulkanischer als auch plutonischer Tätigkeit gewesen. Das allein ist keine Besonderheit des Andenzuges; eine ähnlich verwickelte Geschichte kommt den meisten grossen Kettengebirgen zu. Was aber diesem Gebirge eine erhöhte Bedeutung für die Lösung gewisser allgemeiner Fragen, i. B. nach dem Verhalten der eruptiven Vorgänge zu den gebirgsbildenden verleiht, ist der gewaltige Massstab, in dem hier alle Erscheinungen ausgeprägt sind, ist das Zurücktreten von jungen und zugleich tief hinabsetzenden Versenkungen, wie sie in Europa und Mittelamerika die Faltengebirge jüngerer Entstehung zerstiückelt und dadurch den ursprünglichen Zusammenhang unseren Augen vielfach entzogen haben.

Über 50 Breitengrade und auf einer Längserstreckung von beiläufig $6000 \mathrm{~km}$ verfolgt man die schmale Zone junger Vulkane, die dem Hochgebirge reihen-, gruppenweise oder sporadisch aufgesetzt 
oder ihm eingeptlanzt erscheinen. Aber zwischen den einzelnen Vulkanen oder Vulkanreihen, die scheinbar durch weite vulkanleere Gebiete getrennt werden, stösst man überall auf Spuren junger vulkanischer Tätigkeit; nur weil diese hier zeitlich etwas weiter zurückliegt, nämlich in die Diluvial- und Pliozänzeit fällt, nur weil die älteren Vulkankegel selbst abgetragen und nur ihre Stiele und ihre verstreuten Auswurismassen übrig geblieben sind, erscheint das geologische Bild nicht so geschlossen, wie es in Wirklichkeit ist. Wie der Zusammenhang der Vulkanzone durch die fossilen Eruptionen, so wird ihre Einheitlichkeit durch den gleichartigen Charakter der Gesteinsarten bezeugt, die hier hervorgetreten sind. Liparitische, dazitische und andesitische Typen herrschen bei weitem vor; sie entsprechen, wie gleich gezeigt werden wird, auch der überwiegend granodioritischen Natur der nicht an die Oberfläche gedrungenen Massengesteine. Basische Gesteine von basaltischem Charakter sind fast nur in den allerjüngsten Vulkanbergen verbreitet, sie finden sich auch über die ganze Vulkanzone verteilt, treten aber doch an Masse gegenüber den granodioritischen Gesteinen der früheren Eruptionszeiten durchaus zurück.

Die Vulkanberge stehen zwar grösstenteils auf einer sehr schmalen Zone hinteraneinander gereiht, die im wesentlichen mit den höchsten Erhebungen der Westkordillere zusammenfällt, so dass man oft von einer Vulkanlinie gesprochen hat. An manchen Stellen, so in Ecuador, verdoppelt sich aber die Vulkanlinie, und die Breite der Vulkanzone erreicht gegen $100 \mathrm{~km}$. Der Bereich der vulkanischen Ausbrïche erstreckte sich aber zur Pliozän- und Diluvialzeit über eine Zone von viel beträchtlicherer Breite. Denn die beiden Kordillerenzüge, die das abflusslose interandine Hochplateau Bolivias einfassen, die West- und Ostkordillere, enthalten ebenso wie auch hier und da die dazwischen liegende interandine Hochfläche reichlich Spuren älterer Vulkane, sowohl in der Form von Auswurfsmassen als auch als Stiele, und dies Verhalten erstreckt sich anscheinend bis in die Kordillere Nordargentiniens fort. Die Breite der Eruptionszone dieser älteren Vulkane, wie sie fortan genannt sein mögen, beträgt somit in Bolivia rund $200 \mathrm{~km}$. Im ganzen genommen hat somit die vulkanische Tätigkeit von der Pliozänzeit bis heute einerseits eine beträchtliche Einengung in ihrer Breitenausdehnung erfahren, da die Ostkordillere Bolivias keine jüngeren Vulkane trägt, andererseits ist sie heute auf der westlichen Hauptzone auf lange Strecken unterbrochen, während sie früher fast ganz kontinuierlich 
gewesen zu sein scheint. Diese Einengung ist aber offenbar von einer merklichen $\ddot{A} n$ derung in der Natur des Materials begleitet gewesen, es hat eine Verschiebung nach dem basischen Pole zu stattgefunden, denn den dunklen augit- oder olivinführenden Gesteinen begegnet man unter den Erzeugnissen der älteren Vulkane ebenso selten, wie sie in den jüngeren häufig sind. Entsprechend ihrer effusiven Entstehung sind poröse oder blasige Strukturen in den Gesteinen beider Kategorien weit verbreitet und dienen zu ihrer Unterscheidung von ähnlichen älteren und nicht effusiven Gesténsmassen ebenso gut, wie das vollständige Fehlen der Erzgänge. Es muss als eine Besonderheit des andinen Vulkanismus in jüngerer Zeit hervorgehoben werden, dass die Ausbrïche allgemein auf einzelne, engumschriebene Ausbruchsstellen beschränkt und dabei auch zeitlich engbegrenzt gewesen sind, und dass Massenergüsse in der Form weit ausgedehnter Decken ganz zu fehlen scheinen. Dieser Charakter kommt dagegen in ausgesprochenem Masse den vulkanischen Erzengnissen aus jungtertiärer Zeit zu, die im mittleren und südlichen Patagonien am Ostrande des Gebirges erscheinen und sich von hier weit hinein ins patagonische Tiefland erstrecken. Basaltische Deckenergüsse von zum Teil gewaltiger Ausdehnung bedingen hier vielfach die Form der hochgelegenen 'Tafelberge. Wo sich solche Ergüsse hart an den Rand der Kordillere drängen, mag es scheinen, als griffen sie auf diese über; das ist aber nach allem, was wir wissen, ausgeschlossen.

Weitaus der verbreitetste Typus alttertiärer Eruptivgesteine wird durch die Liparite, Andesite und Dazite dargestellt, die den mesozoischen oder vormesozoischen

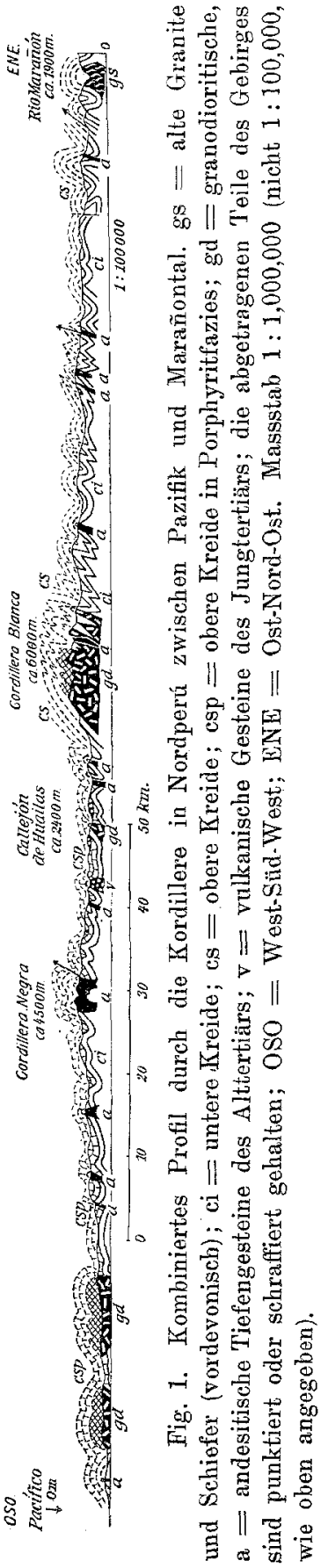


Aufbau der Kordilleren wohl in der ganzen Länge und Breite des Gebirges durchschwärmen, ohne dass sich in erkennbarer Weise effusive Merkmale mit ihnen verknüpfen. Wenn diese Gesteine wirklich bis an die Oberfläche drangen, so ist ibr Ausgehendes jetzt jedenfalls fast überall unsichtbar geworden. Sie treten uns allgemein entgegen als kompakte Gesteine von porphyrischer Struktur in der Form von Gängen, Lagergängen, Stöcken und Lakkolithen. Wohl treten sie vereinzelt bis hart an die Küste des Pacific heran, namentlich in Nordchile und Perú, wo die altkristalline Küstenkordillere sich verschmälert oder verschwindet; sie finden sich auch noch spärlich in den östlichen Ketten der Kordillere, ihr hauptsächlicher Bereich ist aber das eigentliche Hochgebirge (vergl. Fig. 1).

Nicht nur ihrer allgemeinen Verbreitung nach, sondern auch wegen ihrer Eigenschaft als die hauptsächlichsten Erzbringer verdienen diese Gesteine eine besondere Aufmerksamkeit. Man darf wohl behaupten, dass $3 / 4$ aller Erzgänge der Kordillere mit ihrem Empordringen verknüpft und auch örtlich an sie gebunden ist. Das gilt besonders von den Blei-, Silber-, Kupfer- und Zinnerzgängen, während die meisten Goldvorkommnisse, einige Kupferlagerstätten und vereinzelt auch Zinnerz an ältere oder jüngere Tiefengesteine geknüpft sind. Der Zusammenhang zwischen jenen jüngeren Erzgängen und den porphyrischen Gesteinen granodioritischer Natur reicht soweit, dass es wohl kaum einen grösseren Erzdistrilkt gibt, der nicht in oder an einem ausgedehnten Vorkommnis solcher Eruptivgesteine läge, und nur selten gelingt es nicht ohne weiteres, zu einem Erzgange den Erzbringer in nächster Nähe zu finden, denn meist liegt der Erzgang ganz oder teilweise in dem Erzbringer selbst (Fig. 2 Mina Purisima) oder doch in seiner allernächsten Nähe. Diese Erzgänge, die zumeist auch nur einen kurzen Verlauf und oft auch eine wenig regelmässige Gestalt besitzen, verdienen eben die Bezeichnung gebundene im Gegensatz zu den ungebundenen unserer Mittelgebirge, bei denen sich gewöhnlich eine ausgedehnte spaltenartige Erstreckung mit der Unsichtbarkeit des Erzbringers verknüpft findet. 
II.

\section{Gebirgsbildung und Massengesteine in der Kordillere Südamerikas.}

Von

G. Steinmann in Bonn.

\section{Die Kordillere.}

Mit 11 T'extfiguren.

(Fortsetzung.)

An der verschiedenen Natur der Erzgänge werden auch gewisse regionale Differenzierungen des Magmas erkennbar, die sich nur schwer und umständlich, vielleicht auch gar nicht aus den Gesteinen selbst erkennen lassen würden. So konnte bekanntlich STELzner den Nachweis liefern, dass alle bekannten Silber-Zinnerzlager der Kordillere nicht allein an die andesitischen und dazitischen Gesteine der in Rede stehenden Kategorie gebunden sind, sondern dass sie sich auch strenge auf den Zug der Ostkordillere Bolivias beschränken, die an der Ostseite des Titicacasees beginnt und sich bis zur Nordgrenze Argentiniens nach $\mathrm{S}$ erstreckt. Habituell weichen die erzbringenden Gesteine dieses Gebiets nicht von denen $a b$, die sich in ihrer südlichen und nördlichen Fortsetzung finden, und doch ist die Natur der Erzgänge so augenfällig verschieden, und obgleich man in Nordargentinien und in Perú seit dem Erscheinen von Stelzners Arbeit eifrig nach Zinnerz gesucht hat, so hat doch der Zinnerz-Bereich keine Erweiterung erfabren.

Auf die Mannigfaltigkeit der Gesteinsarten dieser Gruppe von Intrusivgesteinen näher einzugehen, ist hier nicht der Platz. Sie bewegen sich zwischen sehr sauren und dann meist auch quarzreichen 
Lipariten einerseits, und Augit- und Hornblendeandesiten von dunkler Farbe andererseits; aber es möge betont werden, dass die letzteren durchgängig recht selten sind, und dass weitaus die Mehrzahl weisslich oder grau bis graugelb gefärbt erscheint, wodurch allein schon ihr saurer Charakter bezeichnet wird.

Es fehlt zur Zeit noch an einem bezeichnenden zusammenfassenden Ausdruck für die geologische Stellung dieser Eruptivmassen. Effusiv, also Ergusssteine sind sie nicht, wie schon betont; höchstens könnten sie hier und da effusive Fortsätze besessen haben, was aber nicht feststeht. Durch ihre Bildung in mässiger Tiefe, durch ihre porphyrische Struktur, durch den Mangel auffälliger Kontaktwirkung sowie durch ihre Ausmaasse gleichen sie den Lakkolithen Nordamerikas und anderer Gegenden, und was sie von ihnen unterscheidet, ist wesentlich nur ihre geologische Form. Diese entspricht nur selten liegenden Linsen, Kuchen oder Platten, wie bei den Lakkolithen, sondern mehr stehenden Linsen oder Platten (Fig. 2) mit allen möglichen untergeordneten Abweichungen davon, die zu den Stöcken hinüberführen. Aber so wie die schwebende Form der nordamerikanischen Lakkolithen mit der allgemein flachen Lagerung der umgebenden Schichtgesteine in Übereinstimmung steht, so wird die vorwiegend stehende Form der andinen Körper aus der meist steilen Stellung und aus der intensiven Faltung der umhüllenden Sedimente verständlich. Sie liegen sehr häufig angenähert konkordant in den aufgerichteten Sedimenten, und auch die Apophysen und Lagergänge nehmen eine mehr oder minder steile Stellung, entsprechend der Schichtneigung, ein (vgl. Fig. 2). Mit Vorliebe stecken sie im Kern oder dicht neben dem Kern der Antiklinalen (Fig. 2). Hiernach glaube ich nicht unpassend zu verfahren, wenn ich die andinen Körper als steile Lakkolithe mit den flachen Lakkolithen des gewöhnlichen. Typus unter der Gesamtbezeichnung Lakkolithe zusammenfasse; fehlt es doch in der Kordillere auch keineswegs an vermittelnden Zwischenformen.

Man weiss schon seit längerer Zeit, dass in den Anden neben den weitverbreiteten Lakkolithgesteinen von porphyrischer Struktur echte Tiefengesteine des granodioritischen Typus von jüngerer Entstehung mehrfach auftreten, aber ibre erstaunlich weite Verbreitung und ihre Bedeutung für die Andengeologie ist mir erst auf meinen letzten Reisen aufgegangen. Wir haben zwei Verbreitungsgebiete dieser "Andengesteine" zu unterscheiden. Das eine zieht zwischen dem Pacific und den Höhen der Westkordillere, also 
an der Westabdachung des Gebirges entlang; es konnte bis jetzt vom nördlichen Perú bis zum mittleren Chile, also über ungefähr 30 Breitengrade mit Sicherheit verfolgt werden. Dieser Abhang der Kordillere setzt sich nun folgendermassen zusammen: 1. aus einer vormesozoischen Grundlage von granodioritischen Gesteinen und Schiefern, 2. aus mesozoischen Gesteinen, grösstenteils eruptiven Ursprungs (Porphyritformation des Jura und der Kreide), 3. aus mittelund jungtertiären Sedimenten an der Küste, 4. aus tertiären Eruptivgesteinen von lakkolithischer Stellung und 5 . aus den in Rede stehenden jüngeren Granodioriten. Eine Unterscheidung dieser letz-

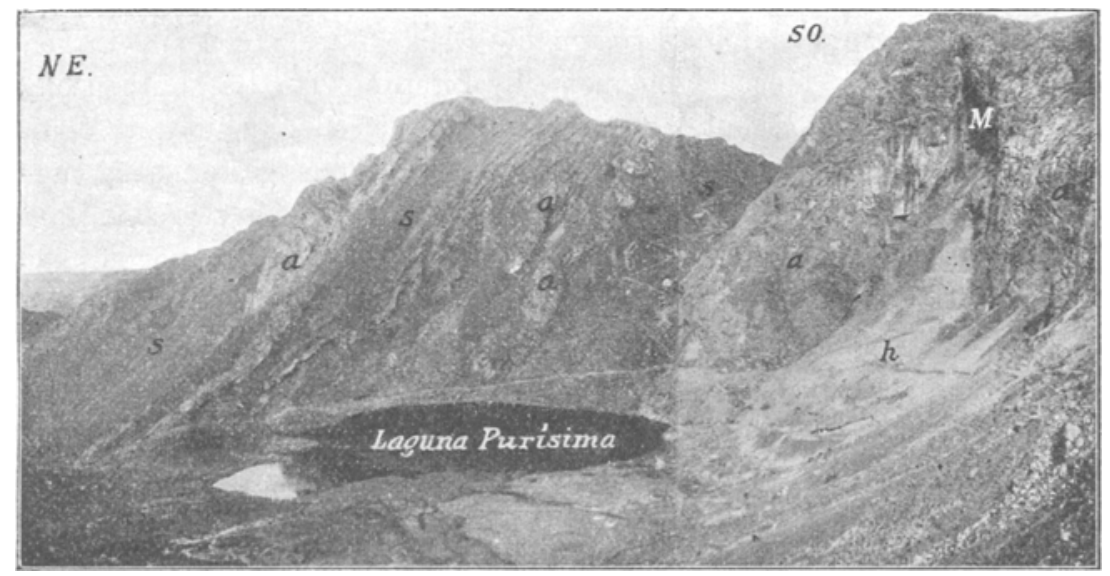

Fig. 2. Blick von Mina Sta. Rosa auf Mina und Laguna Purísima bei Huallanca, N.-Perú. $\mathrm{s}=$ Schiefer und Sandstein des Neokoms; $\mathrm{a}=$ Andesit. Dieser bildet einen grösseren lakkolithartigen Lagergang (rechts), in dem die Mina Purísima (M) mit ihren Halden (h) liegt, ferner schmälere Lagergänge in der Mitte (a) und links. Im Vordergrunde der Glazialsee Laguna Purísima, nach unten abgeschlossen durch Rundhöcker $(\mathrm{R} a)$ aus dem Gestein des rechten Andesit-Lagerganges a. SO $=$ Süd-West.

Phot. Strinmann.

teren von den alten Graniten lässt sich dort überall leicht und mit Sicherheit durchführen, wo die mesozoischen Sedimente vorhanden sind, und da diese den grössten Teil des Kordillerenhanges zusammensetzen und die ältere Unterlage fast nur am Küstensaume und auch hier meist nur in einer schmalen, vielfach unterbrochenen Zone hervortritt, so unterliegt die Feststellung des jüngeren Alters der Andengranodiorite keinen grossen Schwierigkeiten. Ihre durchgreifende Lagerung gegenüber den mesozoischen Sedimenten und die Kon- 
taktveränderungen, die sie an ihnen hervorgerufen haben, bezeichnen sie offenkundig als jüngere Intrusionen.

Die heutige Oberflächenverbreitung der "Andengesteine" auf dieser Zone kann man am besten mit einer locker gereihten Schnur aus verlängerten Perlen vergleichen, die in schwach gewelltem, hier und da verdoppeltem oder vervielfachtem Verlaufe dem Gebirgsabfalle aufliegt. Denn wo die Quertäler tief in den Abhang des Gebirges einschneiden, entblössen sie auf kurzen oder längeren Strecken die Andenbatholithe, and diese verschmälern sich oder verschwinden meist, sobald man von den Tälern aus seitwärts zu den höheren Bergregionen aufsteigt; dann schliesst sich die Hülle über der Kuppel des Tiefengesteins, weil die Erosion die Decke noch nicht abgetragen hat. Man könnte hiernach meinen, die Firstlinie der Batholithenzone laufe angenähert horizontal. Das trifft aber wieder nicht $\mathrm{zu}$, denn in manchen sehr tiefen Tälern erreichen die Andengesteine nur unbedeutende Entwickelung, andernorts steigen sie auch zwischen den Tälern hoch empor. Das erklärt sich nicht aus der mehr oder minder tief reichenden Abtragung, sondern nur aus der Tatsache, dass die Firstlinie auf und abgebogen ist, mit andern Worten, dass das granodioritische Magma an einigen Stellen höher emporgestiegen ist als an anderen. Eine Bestätigung hierfür werden wir bei der Betrachtung der innerhalb der Kordilleren gelegenen Granodiorite finden (s. Fig. 7, 8, S. 24 a. 25).

Die Lagerungsverhältnisse im Dach der Batholithe lassen sich in jener meist ganz und gar wüsten und wasserlosen Gegend auf den Höhen des Gebirges nur schwierig verfolgen, und wir wissen wenig darüber. Aber in der Tiefe der bewohnten Täler und an ihren Abhängen beobachtet man leichter die Grenzregion zwischen Intrusivgestein and Sediment, und da zeigt sich ein verschiedenes Verhalten.

Wo die Spitze eines Batholithen sichtbar wird, wölben sich die Sedimente gewöhnlich antiklinal darüber und die Schichtung läuft mit der Grenzfläche im allgemeinen parallel (Fjg. 3). An den Seiten des Batholithen schleppen die Sedimente nicht selten, und das scheinen diejenigen Stellen zu sein, wo das Dach sich zur Seitenwand hinunterbiegt. Wo dagegen tiefere Teile des Batholithen geschnitten sind und die Seitenwände sichtbar werden, stossen die Sedimente in mehr oder weniger schwebender Lagerung an der steilen oder senkrechten Fläche des Massengesteins ab (Fig. 4), oder sie biegen sich gar schwach oder 
steil geneigt zur Tiefe gegen dasselbe. Letzteres ist aber keineswegs ein Ausnahmefall. Man sieht dieses Einschiessen der Sedimente gegen das Massengestein im Tal des Rio de Copiapó (vgl. das Profil im Atlas der Geologie von Berghaus, Bl. 14), ich fand es bei Lima, wo die Detailstudien Lissóss es bestätigt haben (Fig. 6), und ein

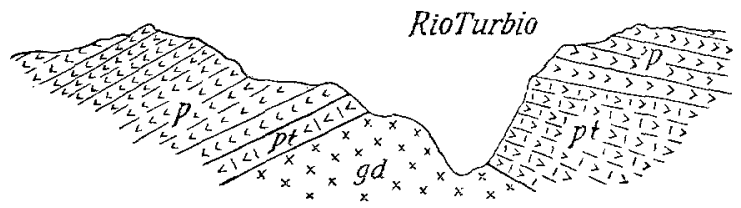

Fig. 3. Querprofil durch das Tal des Rio Turbio in Nord-Chile (nach Donerko). $\mathrm{p}=$ Porphyritische Sedimente des Lias; $\mathrm{pt}=$ dieselben kontaktmetamorph verändert (,tofos"); gd $=$ Granodiorit.

typisches Beispiel dafür gibt beistehendes Profil aus dem Tal des Jequepeteque (Fig. 5). Hier wie im Tal von Copiapó quillt der Granodiorit gewissermassen aus einer zerrissenen und klaffenden Mulde hervor. Bekanntlich hat SALOMON am Adamello ein ähnliches Einfallen der Sedimente gegen den Batholith zu beobachtet und darauf die Bezeichnung Ethmolith gegründet.

Die Tiefengesteinskörper stecken allgemein in den Sedimenten

\section{Cerro San Graviel}
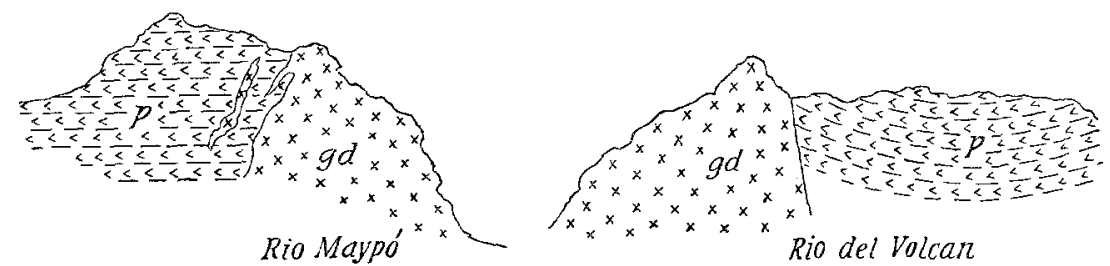

Fig. 4. Profile am Zusammenflass des Rio del Vulcan mit dem Rio Maipó, Mittel-Chile (nach DomErzo); $p=$ porphyritische Sedimente des Mesozoikums ; $g d=$ Granodiorit.

des Jura und der Kreide, die sich zwar zum überwiegenden Teile aus dem effusiven Material der mesozoischen Porphyrite aufbauen, aber doch hinreichend fossilführende Lagen von Kalkstein, Mergel und submarin gebildetem Tuff enthalten, um eine Gliederung zu gestatten. 
So wird es möglich, festzustellen, in welchem Niveau sich der Intrusivkörper befindet, und die Mächtigkeit seines Dachs annähernd zu bestimmen. In der Kordillere von Coquimbo in Nordchile erscheint nach Domeyko bei Carrisal im Tal des Rio Turbio die Spitze des Batholithen unter den fossilführenden Schichten des Lias (Fig. 3); über ihm hat die ganze Mächtigkeit von Jura und Kreide, mehrere (etwa 4-6) Kilometer mächtig gelegen. Im Tal von Copiapó dagegen neigen sich nach meinen Beobachtungen Schichten der mittleren Kreide gegen den Granodiorit von Remolinos; hier kann das Dach etwa nur' ein Drittel so mächtig gewesen sein. Bei Lima sind nach Lissón Kalke von jüngerem Alter als Neokom durch den Diorit kon-

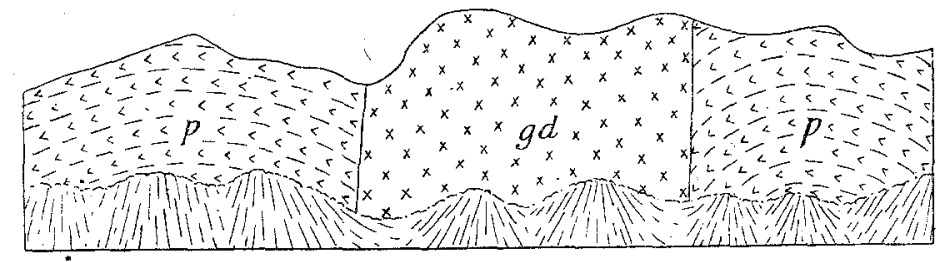

Fig. 5. Profil im Tal des Jequepeteque bei Paypay, N.-Perú. $p=$ Porphyritische Sedimente der Oberkreide; gd $=$ Granodiorit.

taktmetamorph verändert und gegen ihn geneigt; das Dach mag hier die mittlere und obere Kreide umfasst haben, deren Mächtigkeit mindestens auf $3-4 \mathrm{~km}$ zu veranschlagen ist. Nirgends aber habe ich einen Batholithen getroffen, dessen Bedeckung nur gering gewesen sein könnte.

Die aufgeschlossene Breite der Batholithen schwankt natürlich sehr. Massen von nur einigen $100 \mathrm{~m}$ Breite treten in der Kordillere Chiles und Nordperús nicht selten auf. Auf dem Wege von Tacna zum Tacora querten wir einen Batholith von mindestens $8 \mathrm{~km}$ Breite. Im Tal des Rimac aber erstreckt sich der Granodiorit, bei Lima beginnend über fast $50 \mathrm{~km}$ Breite (Fig. 6). Zwar sind ihm mächtige Schollen des durchbrochenen Gebirges eingeschaltet, die zusammen wohl über $10 \mathrm{~km}$ an Breite einnehmen. Immerhin gehen die Ausmaasse dieses Batholithen ungeheuer weit über die Breite der Lakkolithen hinaus, denn diese überschreiten kaum den Betrag von $2-4 \mathrm{~km}$, wo sie eine einheitliche Masse bilden, und nur wo mehrere Eruptionen sich zusammenscharen, werden grössere Beträge beobachtet. Alle diese Maasse sind senkrecht zum Schichtstreichen gerechnet. In der Richtung des Streichens erscheinen aber alle Tiefengesteinskörper er- 
heblich verlängert, und sie stehen in der Tiefe sicher häufig miteinander in Verbindung, was bei den Lakkolithen nicht beobachtet wird.

Innerbalb der Kordillere selbst, mit andern Worten im Osten des Kammes der westlichen Hauptkordillere treten die Andengranodiorite mehr sporadisch auf, aber man kennt sie hier auch über eine gewaltige Strecke. Vom nördlichsten mir bekannten Punkte in Perú, S. Huamachacco $\left(7^{\circ} 50^{\circ}\right.$ s. Br.), bis zum Cerro Payne in Patagonien $\left(51^{0}\right.$ s. Br.), wo Hauthal den südlichsten feststellte, zählt man über 43 Breitengrade; es lässt sich aber nicht mit Sicherheit sagen, ob mit diesen Punkten schon die Enden bezeichnet sind. In den südlichen Teilen des Gebirges lässt sich auch schwer entscheiden, welche Vorkommen dieser und welche der westlichen Zone beizuzählen sind, die eben besprochen wurde.

Zwei Ketten, durch ungewöhnliche Höhe und Geschlossenheit ausgezeichnet, heben sich aus der Kordillere heraus, die Cordillera Blanca in Nordperú zwischen $7^{\circ} 30^{\prime}$ und $10^{\circ} \mathrm{s}$. Br., geographisch gewöhnlich noch der Westkordillere zugerechnet, und die Kordillere des Illimani im Osten des Titicacasees. Beide besitzen eine Anzahl Erhebungen von über $6000 \mathrm{~m}$, und der Huascarán in Nordperú erreicht über $6700 \mathrm{~m}$. Beiden Kordilleren gemeinsam ist das Fehlen von Vulkanbergen, die in der Westkordillere die meisten der höchsten Spitzen bilden; gemeinsam ist ihnen aber auch das Hinaufreichen des Granodiorits in die eisgepanzerten Höhen. Man darf zwar nicht sagen, diese Gebirgszïge bestünden allein oder vorwiegend aus Granit; ihr Kern allein wird von einer langgestreckten, granitischen Injektion gebildet, die der Längsachse der beiden Kordilleren

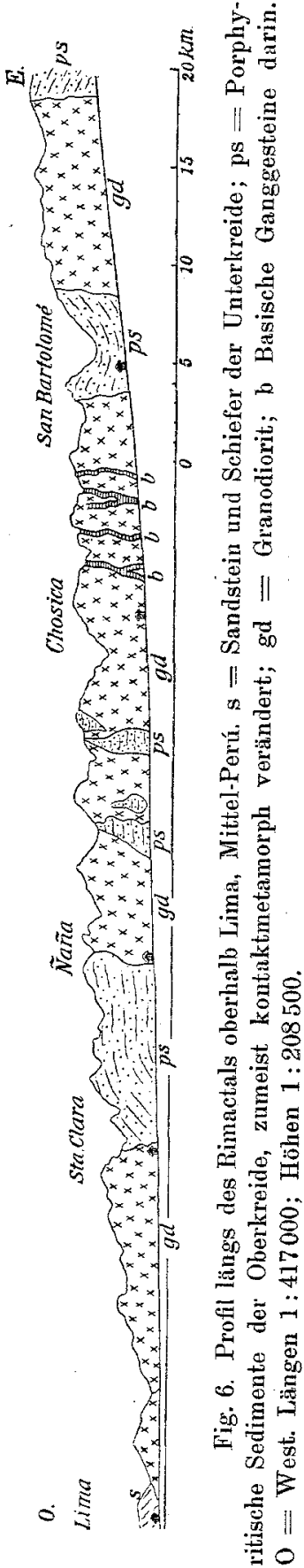


entspricht; aber dieser ist aus der ummantelnden Schieferhülle erst zum kleinsten Teile herausgeschält. Und da nun auch hier das Magma in der Form mehr oder weniger steiler Kuppen zu verschiedener Höhe aufgestiegen ist, so gleicht auch hier die oberflächliche Verbreitung des Massengesteins einer Perlschnur, die von kontaktmetamorphen Schiefern eingefasst wird.

Das junge, postkretazische Alter der Granodiorite steht für die Cordillera Blanca unbezweifelt fest. Denn fast überall steckt das Massengestein in einer Hülle von Schiefern, in denen tithonische oder neokome Ammoniten reichlich vorkommen, und selbst die kon-

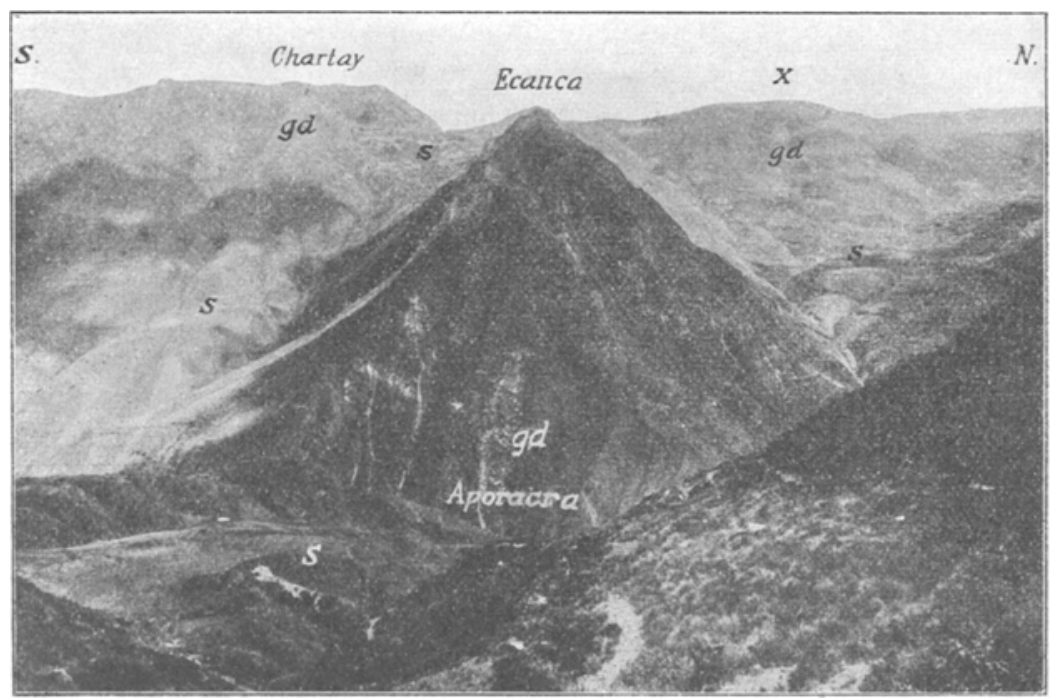

Fig. 7. Blick von Yantacón gegen W auf die Granodioritbatholithe zwischen Herahuayoc und Corongo, Nordende der Cordillera Blanca in N.-Perú. s = Schiefer und Sandsteine des Tithons und der Unterkreide, z. gr. T. kontaktmetamorph veründert; gd = Granodiorit-Batholithe. Von diesen reihen sich Chartay, Ecanca (durch Aporacra fast ganz verdeckt) und $x$ in der Richtong der Faltenachse SO-NW) aneinander. Aporacra, gegen $O$ davor liegend, verbreitert sich rasch nach unten.

Phot. Steinmann

taktmetamorphen Schiefer enthalten noch gut erkennbare Reste. Die Sedimente der Kordillere des Illimani bestehen dagegen nur aus Schiefern und Quarziten des Untersilurs und Sandschiefern des Devons, und daher hat man die darin injizierten Granite bisher unbedenklich für paläozoisch erklärt. $\mathrm{Wir}$ werden aber sehen, dass sich auch für diese Granite ein jugendliches Alter durchaus wahrscheinlich machen lässt. 
Die Granodiorite dieser beiden Kordilleren weisen nämlich einen gleichen und sehr einförmigen Habitus auf. Sie sind hell, weiss bis grau gefärbt, grobkörnig, stellenweise porphyrisch, untergeordnet auch fluidal struiert bis zur Ausbildung von Augengneis. Hier und da treten hornblendereiche und quarzarme Randbildungen auf, scheinen aber nirgends erheblichen Umfang zu erreichen. Der Durchschnittstypus gleicht den hellen Graniten unserer Mittelgebirge und der Alpen mit häufiger Hinneigung zu porphyrischer Struktur (Fichtelgebirgsgranit, Kammgranit der Vogesen, Schluchseegranit im Schwarzwald, Zentralgneis der Tauern). Nur im Inneren

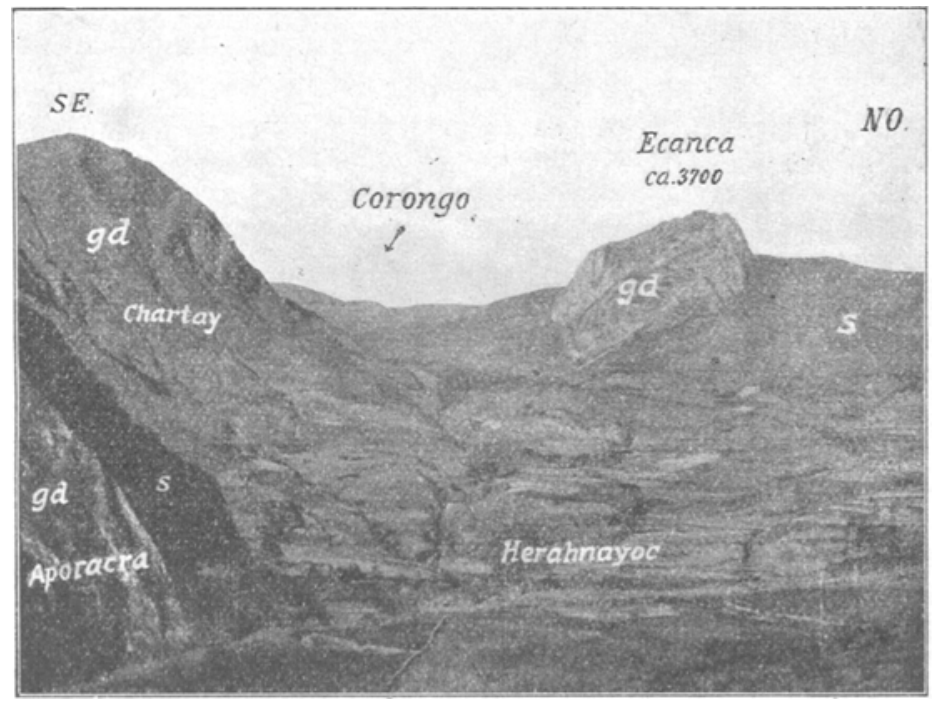

Fig. 8. Blick auf die Granodioritbatbolithen zwischen Corongo und Herahuayoc, N.-Ende der Curdillera Blanca, N.-Perú. s = Schiefer und Sandstein des Tithons und der Unterkreide, grösstenteils kontaktmetamorph; $g=$ Granodiorit. Ecanca ragt als steiler Kegel aus dem Schieferlande empor. $\quad$ NO $=$ Nord-West.

Phot. Schlagintweit.

des Massivs der Cordillera Blanca traf ich wenig Muskowit beigemischt, sonst herrscht Biotit allein, dem sich zuweilen Amphibol zugesellt. Aplit- und Pegmatitgänge sind überall häufig, auch basische Ganggesteine treten manchmal hervor, über ihre Zusammensetzung ist aber noch so gut wie nichts bekannt. Während mit den Granodioriten der westlichen Zone häufig Kupfer- und Goldgänge verknüpft erscheinen, erweisen sich diese beiden Gebiete des jüngeren Granits als recht steril (man kennt nur an einigen Stellen Goldquarzgänge, aus 
denen z. B. das Waschgold im Rio Tablachaca, Nordperú, und das von Chuquiaguillo bei La Paz stammt); sie unterscheiden sich hierdurch auch von den alten (vormesozoischen) Graniten, die im $O$ der Kordillere verbreitet und fast überall als goldführend bekannt sind.

Die Sedimenthülle der Andengranodiorite ist, wie bemerkt, in beiden Kordilleren nur zum geringen Teil entfernt. Darauf beruht u. a. auch die Erscheinung, dass die kontaktmetamorphen Hïllschiefer und Sandsteine im Dach und an den Seiten der Granodioritkörper

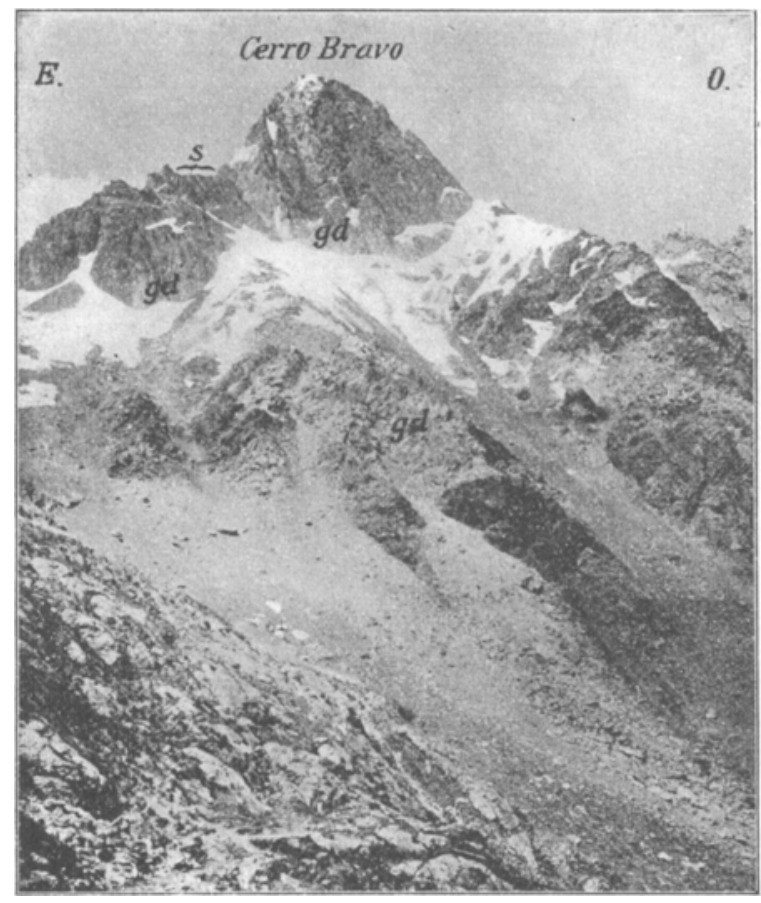

Fig. 9. Einklemmung von kontaktmetamorphem, steil nachW fallendem Nenkomschiefer (s) in den Granodiorit (gd) der Cordillera Blanca, N.-Perú. Vom Yanganucopass fea $4700 \mathrm{~m}$ ) aus gesehen. $O=$ West. Phot. Steinmann. ausgedehnte Flächen einnehmen, so dass man tagelang nicht aus Chiastolithschiefern

herauskommt. Dort, wo die Cordillera Blanca an ihrem Nordende an Höhe beträchtlich verliert und die Firstlinie der Granitmasseimmer tiefer sinkt, schliessen die kontaktmetamorphen Hüllschiefer über ihr zusammen, und nur an einzelnen Stellen ragen die höchsten Teile des Intrusivkörpers daraus hervor. Diese erscheinen dann hier in der Form isolierter Kegelberge, um die sich die Hüllschiefer mantelförmig herumlegen und zwischen denen sie tief hinabreichen. Da die Erosion die Schiefer leichter entfernt als den Granit, so überragen die Granitberge ihre Umgebung als auffallende Klötze mit steilem Abfall (vgl. hierzu Fig. 7, 8). Wo ein solcher Granitkegel hart am Tale steht und etwas tiefer angeschnitten ist als gewöhnlich, sieht man auch, wie er sich nach unten verbreitert (vgl. Fig. 7), 
und man gewinnt die Überzeugung, dass mehrere benachbarte Kegel in der Tiefe zu einer gemeinsamen Masse zusammenfliessen. Zu derselben Deutung führt die Betrachtung der höheren Kammregion des Gebirges (vgl. Fig. 9). Hier sieht man öfters die metamorphen Hüllschiefer sich von oben her in die geschlossene Granitmasse einkeilen. Solche Vorkommnisse ergänzen sich mit dem eben geschilderten Bilde zu der Vorstellung, wonach die Oberfläche des Granitmassivs gewissermassen den Faltenbau wiederspiegelt, d. h. aus steilen Parallelkämmen besteht, die unter den Antiklinalen des Hüllsediments verlaufen und sich mit ihnen erheben, während unter den Sedimentmulden das Massengestein ebenfalls muldenartig herabsinkt. Entsprechend dem wellenförmigen Verlaufe der Firstlinien der Antiklinalen steigt auch die Firstlinie des darin injizierten Granits auf und nieder and erzeugt daher das Bild einzelner Kegel, die in der Streichrichtung der Falten hintereinander geschaltet unter diesen emporsteigen.

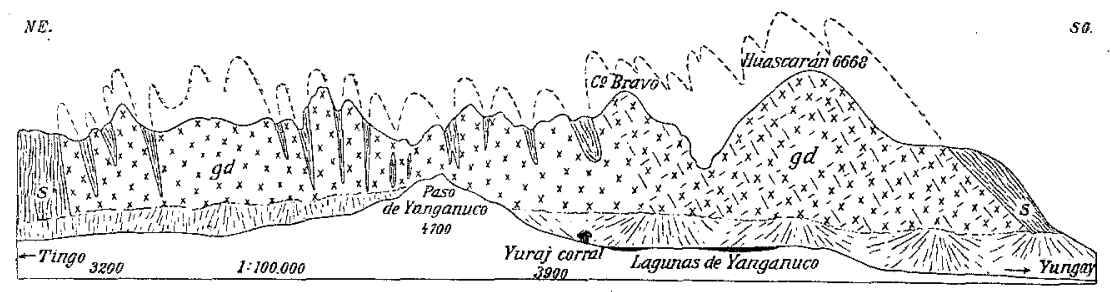

Fig. 10. Profil durch die Cordillera Blanca in der Breite von Yungay, N.-Perú stark überhöht. $\mathrm{s}=$ kontaktmetamorphe Schiefer und Sandsteine des Tithons und der Unterkreide; gd $=$ Granodiorit, im W. parallel der Grenzfläche gebankt.

Die Falten der Kordillere sind fast durchgängig gegen $O$ übergeneigt, und sehr häufig steigert sich die Faltung zur Überschiebung nach der gleichen Himmelsrichtung (Fig. 1, S. 16). Diese Einseitigkeit der Bewegung macht der Granit mit. Auf dem Westabhange der Cordillera Blanca beobachtet man fast allgemein ein Abfallen der Hüllschiefer vom Granit mit of wenig steilem Einfallen gegen W, auf der Ostseite dagegen tauchen die Schiefer mit vorwiegend steilem Westfallen unter den Granit unter, und ebenso neigen sich die zahlreichen Einspitzungen des Schiefers im Granit auf dem Ostabhange des Gebirges fast allgemein gegen. W (vgl. Fig. 10).

Hiernach besteht an der oberen Grenze der Granitintrusivkörper gegen die Hüllschiefer ein konkordanter Injektionsverband, wie ich es nennen möchte, wenigstens im grossen und ganzen. Im einzelnen verzweigt sich aber das Massengestein auch in der Form 
vielgestalteter Fortsätze gangartig in der Sedimenthülle, und die Granitgänge queren ebenso häufig die Schichten als sie sich zwischen sie einschieben.

Für die Kordillere des Illimani lässt sich ein nachmesozoisches Alter nicht so offenkundig erweisen, wie für die Cordillera Blanca. Dafür spricht aber ausser der vollständigen Übereinstimmung im Gesteinscharakter auch die gleiche Art des Auftretens, i. B. das Aufsteigen bis zu Höhen von über $6000 \mathrm{~m}$, wogegen die vormesozoischen Granite allgemein in der Tiefe bleiben, da sie die Unterlage der überall mächtigen paläozoischen Serie bilden. Zudem traf ich an einer Stelle in der Nähe des Illimani einen Gang von Granitporphyr im Kreidesandstein, was nur im obigen Sinne eines jüngeren Alters des Granits gedeutet werden kann. Da ferner die intensive Faltung der paläozoischen wie der mesozoischen Sedimente in die Tertiärzeit fällt und sich der Granit der Kordillere des Illimani nach allem, was man

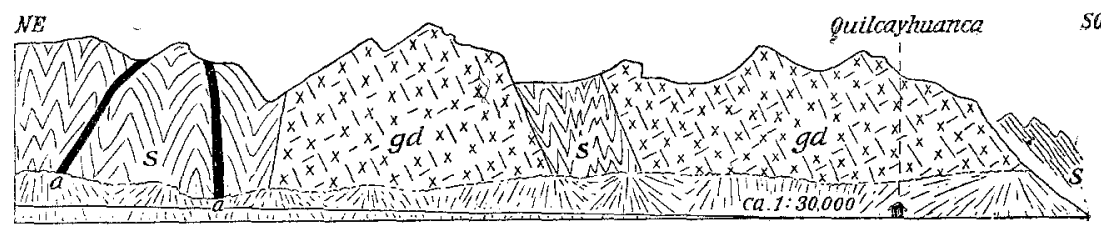

Fig. 11. Profil durch die Cordillera Blanca entlang der Quebrada de Quilcayhuanca bei Huaraz (N.-Perú), überhöht. $s=$ kontaktmetamorphe Schiefer und Sandsteine des Tithons und der Unterkreide; gd = Granodiorit; $\mathfrak{a}=$ Andesit. $\mathrm{SO}=$ Südwest. Bankung des Granodiorits parallel mit der Schieferkontaktfläche im Westen.

darüber weiss, diesen gegenüber ebenso verhält wie der der Cordillera Blanca, so kann ein berechtigter Zweifel am tertiären Alter der Injektion nicht wohl aufkommen.

Obgleich manche Täler der Cordillera Blanca mebr als $3000 \mathrm{~m}$ tief in das Gebirge einschneiden, wird hier doch unter dem Granodiorit kein anderes Gestein sichtbar. Fast ebenso tief reichen die Taleinschnitte auf dem Westabhange der Kordillere hinab, und auch diese erschliessen für gewöhnlich das Liegende des Massengesteins nicht. Wir dürfen also die vertikale Mächtigkeit der Batholithe auf mindestens $3-4 \mathrm{~km}$ veranschlagen. Es wäre aber unrichtig anzunehmen, die Batholithe setzten überall als einheitliche und geschlossene Masse in die unendliche Tiefe fort. Schon in dem Profile durch die Quebrada de Quilcayhuanca (Fig. 11) schiebt sich zwischen zwei Granitmassen eine breite Schiefermasse ein, von der sich nicht sagen lässt, 
ob sie nicht etwa in die Tiefe fortsetzt und sich hier vielleicht gar verbreitert.

Der Taleinschnitt des Rio Rimac oberhalb Lima (Fig. 6, S. 22) zeigt aber noch deutlicher, dass in der Tiefe der Batholithe beträchliche Massen der vom Granodiorit durchbrochenen Gesteinskomplexe erhalten sind. Denn hier erscheinen inmitten der scheinbar einheitlichen Granodioritmasse mehrfach fremde Gesteine nicht allein als Schollen von etwa $1-2 \mathrm{~km}$ Breite, sondern auch mächtige Klötze, die fast $10 \mathrm{~km}$ Breite erreichen. Diese Massen sind durchgängig hochgradig verändert und werden am Kontakte von Pegmatitgängen durchschwärmt, sind also zweifellos bei der Injektion des Granits vorhanden gewesen. Anfangs hatte ich sie für basische Ausscheidungen des Granodiorit-Magmas selbst gehalten, aber das sind sie keinesfalls. Die untersuchten Proben erweisen sich vielmehr als umgewandelte Porphyritgesteine des Mesozoikums, und in der östlichen Scholle bei S. Bartolomé haben sie ihren ursprünglichen Charakter auch vollständig bewahrt. Es werden also in der Tiefe der Batholithe zuweilen gewaltige Stücke der durchbrochenen Rinde sichtbar, und dieses Verhalten erscheint vielleicht nur deshalb als Ausnahme, weil die vorhandenen Aufschlüsse nur selten einen so tiefen Einblick gestatten wie im Rimactale. An dem Granodiorit zeigen sich aber auch hier keine Erscheinungen, die darauf schliessen liessen, dass grössere Mengen der vorhandenen Rinde vom Magma eingeschmolzen und assimiliert worden seien. Denn in diesem Falle müsste eine reichliche Beimischung solch stark basischen Materials, wie es die Decken, Tuffe usw. der mesozoischen Porphyrite darstellen, dem Granodiorit auch eine auffällige basische Lokalfärbung erteilt haben. Ausser basischen Randbildungen von geringer Ausdehnung sieht man aber derartiges nicht. Alle mit den Granodioritbatholithen verknüpften Erscheinungen deuten vielmehr darauf hin, dass das Magma in die gefalteten oder zerstückelten Sedimente injiziert worden ist.

Das Alter der effusiven Eruptivmassen der Kordillere lässt sich auf doppelte Weise ziemlich genau fixieren. Vulkanische Tuffe beteiligen sich, wie wir gesehen haben, an der $\mathrm{Zu}$ sammensetzung rezenter, diluvialer und jungtertiärer Sedimente, und die letzteren reichen, soweit wir das beurteilen können, wohl kaum weit hinter die Pliozänzeit, vielleicht noch bis ins Miozän zurück. Auch schmiegen sich alle vulkanischen Bildungen entweder dem heutigen Relief an oder doch einem früheren insofern ähnlichen, als es uns das Gebirge schon von tiefen Tälern durchschnitten oder von breiten 
Senken durchsetzt zeigt. Für die Intrusivmassen der andesitischen Lakkolithe und granodioritischen Batholithe lässt sich nur allgemein behaupten, dass sie älter sind, denn sie werden durchgängig von jedem erkennbaren Relief beschnitten und ihre heutige Entblössung setzt Abtragungen in einer Mächtigkeit von Kilometern und eine tiefe Durchfurchung des Gebirges $\mathrm{nach}$ ihrer Bildung voraus, sie sind also wohl jedenfalls älter als Pliozän oder Miozän.

Bedauerlicherweise sind bis heute irgendwelche fossilführende Absätze der mittleren und älteren Tertiärzeit in der Kordillere so gut wie ganz unbekannt, und das steht einer genaueren Altersbestimmung der Intrusiva hindernd im Wege. Einen brauchbaren Anhalt gewinnen wir nur in dem Verhalten der Intrusiva zur Faltung.

Allen jüngeren Eruptivmassen, die wir bis jetzt betrachtet haben, gehen Erscheinungen ab, die auf die Wirkung eines allgemein verbreiteten Gebirgsdrucks zurückgeführt werden müssen. Nur ganz lokal treten an den Granodioriten oder Andesiten Verruschelungen auf; sie sprechen für ein abgeschw ächtes Fortsetzen gebirgsbildender Vorgänge in jüngere Zeiten. So können wir behaupten, die Eruptivmassen sind allgemein jünger als die Hauptfalt ung der Kordillere. Diese hat nun auch die jüngsten marinen Sedimente der Kreide mit ergriffen, so in Perú und Kolumbia das Senon. Die Batholithe von Granodiorit und die Lakkolithe andesitischer Gesteine treten zwar für gewöhnlich nur mit Gesteinen des Tithons und der Unterkreide (Neokom-Gault) in Berührung, und das ist ja auch zu erwarten bei ihrer geologischen Stellung als Intrusivgesteine. Doch durchsetzen die andesitischen Lakkolithe an einigen Stellen auch Gesteine der Oberkreide, und dasselbe trifft für einige Granodioritvorkommnisse zu. Somit kann man das Alter der Intrusivgesteine nach rückwärts durch die postkretazische Faltung, nach vorwärts durch das Jungtertiär begrenzen und ist berechtigt, sie allgemein als alt- oder mitteltertiär, sowie jünger als die Hauptfaltung zu bezeichnen.

Das Empordringen von Intrusivgesteinen im Anschluss an eine vorausgegangene Faltung eröffnet hier wie in so vielen anderen Fällen die Frage nach den ursächlichen Beziehungen zwischen beiden Erscheinungen. Eine einseitige, gegen 0 gerichtete Faltung beherrscht die Anden, und wie an den Granodioriten der Cordillera Blanca gezeigt werden konnte, fügen sich ihre Massen in einem langen, schmalen Zuge diesem Faltungsrabmen ein. Das führt notgedrungen zu der Vorstellung, dass der Raum für die Intrusivmassen im wesentlichen durch die Faltung selbst geschaffen wurde. Weder die andesitischen 
Lakkolithe noch die granodioritischen Batholithe haben sich in dem gefalteten Gebirge durch Aufschmelzen ihren Platz geschaffen, sondern sie füllen offenbar vorher gebildete Höhlungen, was nicht ausschliesst, dass der Raum, in dem sie einmal eingelassen, sich dann noch etwas erweitert habe. Schon die kontaktmetamorphe Umwandlung der Hüllschiefer kommt einer Verdichtung ihres Mineralbestandes und damit einer Erweiterung des Raumes gleich, den das Magma ausfüllen kann. Wie weit letzteres dabei auch noch aktiv beteiligt ist, mag vorläufig unerörtert bleiben.

Diese Vorstellung, wonach der Faltungsvorgang die Hohlräume geschaffen hat, in die das Magma eindringen konnte, entspricht nach meiner Auffassung am besten den Verhältnissen, die wir in den beiden höchsten granodioritischen Kordillerenzügen Perú-Bolivias beobachten. In der Cordillera Blanca wie in der Cordillera des Illimani sind die Sedimente (im ersten Falle die Kreide, im anderen die paläozoischen und die Kreide) im Verhältnis zu ihrer Umgebung besonders hoch aufgefaltet. Man kann diese Überhöhung in beiden Fällen auf etwa $3 \mathrm{~km}$ veranschlagen. An dieser Auffaltung hat aber das Liegende, im besonderen das vorsilurische oder vorkambrische Grundgebirge, nicht mit teilgenommen. Es liegen aber auch keine Anzeichen dafür vor, dass dies Liegende vom Magma eingeschmolzen sei, und ebensowenig können wir uns vorstellen, es sei ursprünglich mit emporgefaltet und dann etwa durch Absinken von der darüber gespannten Sedimentdecke in grosse Tiefen versenkt worden. Denn bei der intensiven Faltung und der weitgehenden Verfaltung, die an anderen Stellen zwischen Grundgebirge und Sedimentdecke beobachtet wird, könnte dieser Vorgang unmöglich zu einem glatten Ablösen der Unterlage geführt haben. Viel plausibler erscheint dagegen die Annahme, dass das Grundgebirge nicht entsprechend hoch mit aufgefaltet, sondern in der Tiefe zurückgeblieben ist, so dass zwischen ihm und der aufgefalteten Decke ein antiklinaler Hohlraum oder eine Reihe kleinerer Räume entstanden. Wurde durch einen solchen Abstau das Liegende entlastet und zugleich beim Nachlassen des Seitendrucks schollenartig zerstïckelt, so war dem Magma der Zutritt aus der 'Tief'e gebahnt, und es konnte in die abgestauten Hohlräume eindringen. Hiernach müssten wir in der Tiefe der Batbolithe die zurückgebliebenen Teile als dislozierte Schollen anzutreffen erwarten, und diese Voraussetzung scheint denn auch durch Fälle wie im Rimactale (S. 22) bestätigt zu werden. Bekanntlich ist man auch in einem schwach gewellten Faltengebirge wie dem Schweizer Jura zu der Vorstellung gelangt, dass durch die Faltung eine relativ wenig mächtige 
Oberlage von einer nicht mit aufgefalteten Unterlage abgeschert worden ist, ein Vorgang, mit dem sich die Bildung von antiklinalen Hohlräumen naturgemäss verknüpft. Wesentlich ähnlich darf man sich wohl den Faltungsprozess in den Kordilleren vorstellen, nur insofern abweichend, als er sich in viel grossartigerem Massstabe abspielte, eine weit mächtigere Oberlage ergriff und daher auch eine viel beträchtlichere Entlastung herbeiführte, die hier im Gegensatz zum Faltenjura den Auftrieb des Magmas in die Hohlräume der Oberlage gestattete. Aus dieser Vorstellung, wonach Hohlräume von zum Teil beträchtlicher Länge und erheblicher Mächtigkeit eine häufige, vielleicht gesetzmässige Begleiterscheinung der Faltung bilden, wird auch eine andere Erscheinung leicht begreiffich, die die Anden mit dem Juragebirge teilen: die nachträglichen Einbrüche. Wo die Hohlräume nicht durch magmatische Intrusionen ausgestopft wurden, konnten sie früher oder später durch Einbruch der Decke ausgefüllt werden, und so erklärt sich im Jura wie in den Anden das häufige Auftreten von Längsund Querbrüchen, die offenbar jünger sind als die Faltung. Bemerkenswerterweise fehlen aber diesen Brüchen in beiden Gebieten solche Begleiterscheinungen so gut wie ganz, die auf ein Fortsetzen in sehr grosse Tiefen deuten, vulkanische Eruptionen, Erzgänge, Verkieselungserscheinungen, Thermen und dergl., wie sie z. B. an den grossen Rheintalspalten haften. Wenn auch viele Dislokationen der Anden, die auf den ersten Blick einfache Brüche zu sein scheinen, sich bei genauerer Kenntnis des Gebirges als Überschiebungen erweisen, so ist doch die allgemeine Verbreitung von Brüchen im Bereiche der Kordillere durch die verschiedensten Beobachter sicher gestellt.

Trotz der zahlreichen klaren Aufschlüsse im Gebirge lässt sich auf eine andere wichtige Frage nur schwer eine Antwort geben, wie weit nämlich den magmatischen Vorgängen eine aktive Rolle bei der Gebirgsbildung zugeschrieben werden darf. Man könnte ja geneigt sein, die Granite der Cordillera Blanca und der Illimani-Kette für die erhöhte Auffaltung dieser Ketten überhaupt verantwortlich zu machen. Dagegen sprechen aber viele Tatsachen, von denen nur eine besonders wichtige hervorgehoben werden möge, die schon betont wurde. Die Granodioritmassive fügen sich in ihrer. Masse und besonders axich mit ihrer Oberfläche in den allgemeinen, einseitig gegen $O$ bewegten Faltenbau des Gebirges ein, und wo sie fehlen; scheint der Aufbau nicht wesentlich anders zu sein als dort, wo sie vorhanden sind. Eine von unten her wirkende, hebende oder stossende Kraft sollte sich aber doch anders äussern, als eine seitlich faltende, oder 
ihre Wirkung kann nur gering sein. An einigen Orten verknüpft sich wohl mit dem Auftreten der Andesit-Lakkolithe eine lokale Rückfaltung auf der Westseite des Intrusivkörpers. Ich war daher auch geneigt, diese Abweichungen auf Kosten der Intrusion selbst zu setzen, und vielleicht trifft diese Deutung auch in manchen Fällen zu. Wenn man aber sieht, wie die Achsen der Falten im Gebirge keineswegs immer geradlinig, sondern häufig geschlängelt verlaufen, und wie an den nach $W$ konvexen Stellen sich die Schichten zurückbiegen und steil nach $O$ fallen, ohne dass eine grössere Eruptivmasse in der Nähe sichtbar wird, so gerät man in Zweifel, ob jene Deutung zulässig ist.

Wir haben die intrusiven Gesteine in granodioritische Batholithe und andesitische Lakkolithe unterschieden. Jene bilden grössere Massive von körnigem Gestein, diese kleinere Kuchen, Linsen und Stöcke von porphyrisch struiertem Gestein; mit jenen verknüpfen sich nur spärliche und einförmige Erzgänge, diese sind die Bringer der mannigfaltigsten epigenetischen Erzvorkommnisse. Beide sind offenbar in unmittelbarem Anschluss an die Hauptfaltung des Gebirges injiziert worden, aber die granodioritischen Tiefengesteine scheinen zuerst, die andesitischen Lakkolithgesteine etwas später entstanden zu sein. Wir dürfen hiernach annehmen, dass das einheitliche Magma, dem beide entstammen, sich gespalten hat in ein Teilmagma, das, wohl an Masse überwiegend, in gewaltige Hohlräume eindrang, nur wenig mineralbildende Stoffe einschloss und daher wenig Erzgänge erzeugte, und in ein anderes, an Masse zurücktretendes, das reich an mineralbildenden und erzartigen Stoffen war, und das nicht in der Form grösserer Massive erstarrte, sondern sich in kleinere erzbringende Körper verzettelte. Waren diese beiden Teile auch nach ihrer Zusammensetzung und Zeitfolge verschieden, ebenso wie die Räume, die sie erfüllten, so kommt ihnen doch insofern eine ganz gleiche geologische Stellung $z u$, als sie offenbar in wesentlich gleichem Abstande von der Oberfläche zur Erstarrung gelangt sind. Denn zahlreiche Beobachtungen führen zu dem Ergebnis, dass die Batholithe keineswegs allgemein in tieferen geologischen Niveaus stecken als die Lakkolithe. Zumeist wird die Hülle bei den einen wie bei den anderen aus dem Tithon oder aus dem tiefsten, sehr mächtigen Gliede der Kreide gebildet, den Schiefern und Sandsteinen des Neokoms, deren Alter durch tierische und pflanzliche Fossilien an zahlreichen Punkten festgestellt ist. Zuweilen treten auch ältere Horizonte noch mit in die Umhüllung ein, aber gewöhnlich nur in beschränkter Ausdehnung. Es gibt aber auch Lakkolithe, die in der 
Trias stecken und Granodiorite, die Gesteine der Oberkreide durchbrochen haben. Deshalb dürfte der Unterschied zwischen körnigen Batholithgesteinen und porphyrischen Lakkolithgesteinen in der Kordillere nicht auf der grösseren oder geringeren Tiefe, in der sie erstarrt sind, beruhen, sondern wohl nur auf langsamerer oder rascherer Abkühlung, entsprechend dem durchgängig beträchtlichen Unterschiede ihrer Massen. "Tiefengesteine" sind sie beide, die einen körnige, die anderen porphyrische.

Auf die intrusive Tätigkeit der älteren oder mittleren Tertiärzeit ist im Jungtertiär die effusive gefolgt, und diese dauert stark eingeschränkt bis heute an. Alle Beobachtungen an jungtertiären Absätzen deuten darauf hin, dass sie sich auf einem stark akzentuierten Relief bildeten, dass die vorher gefaltete Masse der Anden jetzt auch als durchtaltes Gebirge emporragte, in dessen Becken und Tälern Absätze von Geröllen, Sanden, Tuffen usw. entstanden. Von den morphologischen Verhältnissen des Andengebietes in altund mitteltertiärer Zeit wissen wir wenig, die Anzeichen alter Fastebenen in bedeutenden Höhen des Gebirges (3000-4000 m) gestatten aber den Schluss, dass die spätere Form des Hochgebirges noch nicht vorhanden war und dieses erst durch eine beträchtliche regionale Hebung, etwa zu Beginn des Jungtertiärs entstand. Vielleicht darf man in dieser Heraushebung auch die Ursache für den Übergang von der intrusiven Tätigkeit zur explosiven suchen. Wir können uns vorstellen, dass mit der Emporhebung der Andenmasse dem Reste des Magmas Gelegenheit geboten wurde, dem vadosen Wasser zu begegnen, und dass hierdurch eine phreatische Phase, wie Suess sagen würde, eingeleitet wurde. Dass diese weiterhin andauerte und bis zur Gegenwart fortbesteht, findet seine Erklärung in der Fortdauer gebirgsbildender Vorgänge bis heute. Denn die jungtertiären Sedimente erscheinen in den verschiedensten Teilen der Kordillere stark disloziert, häufig ganz steil aufgerichtet, offenbar durch Faltung, andernorts von beträchtlichen Verwerfungen durchsetzt, selbst alt. quartäre Tuffe und Geröllmassen sind hier und da noch auffallend geneigt. Faltung und Einbruch haben also fortgedauert, wenn auch nicht mit der früheren Intensität und in der allgemeinen Verbreitung wie ehedem. Aber auch die allgemeine Hebung der Andenmasse ist noch weitergegangen. Hierfür sind neben der hohen Lage der Fastebenen verschiedene Tatsachen von Bedeutung. Pflanzenführendes Jungtertiär liegt in gestörter Lagerung auf dem Cerro de Potosí in über $4500 \mathrm{~m}$ Höhe. Diese Flora ganz moderner Pflanzentypen kann aber nicht in der Puna gewachsen sein, sie setzt eine erheblich, etwa 
$2000 \mathrm{~m}$, geringere Meereshöhe für ihr Gedeihen voraus. Ebenso liegen bei Sinsin zwischen Cajamarca und Hualgayoc in Nordperú jungtertiäre Kohlen unter geneigten Andesittuffen in einer Höhe von über $3500 \mathrm{~m}$; auch diese können nicht unter den Bedingungen der kalten Höhen, sondern nur in tieferem Niveau entstanden sein. Ferner zeigen die Meeresterrassen der Westküste eine Hebung in allerjüngster Zeit an. Da sie aber zwischendurch ganz aussetzen, wo sie bei einer gleichmässigen Hebung vorhanden sein sollten, wie in der Gegend von Lima, so ist die Hebung bis in die jüngste Zeit ungleichmässig gewesen, und Seitendruck und Faltung haben ihren Anteil daran gehabt.

Es laufen also in der Kordillere während der Tertiärzeit zwei Vorgänge enge miteinander verknüpft einher, Faltung des Gebirges und Aufstieg des Magmas. Der Hauptfaltung zur älteren oder mittleren Tertiärzeit schliesst sich die Injektion von Tiefengesteinen in der ganzen Länge des Hauptzuges des Gebirges an; mit der ausklingenden und örtlich eingeengten Faltung zur jüngeren Tertiärzeit (bis heute) lokalisieren sich die magmatischen Vorgänge und schwächen zugleich ab bis zum fast völligen Verschwinden. Dabei findet keine Verlagerung des Magmaherdes statt, sondern die vulkanischen Ereignisse der jüngeren Tertiärzeit vollziehen sich auf einer verschmälerten Zone des alttertiären Intrusivstreifens, und die diluvialen und heutigen Vulkane fallen ebenso wie die vulkanischen Nachwirkungen (Thermen) nur an einzelnen Stellen aus einer Linie heraus, die im wesentlichen durch den westlichen Hauptkamm des Gebirges (Cordillera de los Andes) bezeichnet wird. Diese Linie bildet aber zugleich auf weite Strecken annähernd die Mittellinie für den Bereich, der von granodioritischen Batholithen and von andesitischen Lakkolithen durchschwärmt wird. Darin liegt die geologische Bestätigung für die lithologisch erhärtete Tatsache der Einheitlichkeit und Blutsverwandtschaft der tertiären Massengesteine in der Kordillere; aus der Erschöpfung der magmatischen Tätigkeit erklärt sich der von STüBEL betonte monogene Charakter der jüngeren Vulkanberge.

Die südamerikanische Kordillere bietet wohl das grossartigste und klarste Beispiel aus jüngerer Zeit für die Verknüpfung eruptiver Vorgänge mit der Aufrichtung eines gewaltigen Faltengebirges. Aber sie heften sich nirgends an die späteren Einbrüche des Gebirges; weder die Senkungsfelder zwischen den auseinander tretenden Ästen der Kordillere im Norden, noch die Verwerfungen im geschlossenen Hochgebirge, noch endlich der langgestreckte Abbruchsaum der pazifischen Küste zeigen Begleiterscheinungen vulkanischer Natur. 